ISBN 978-93-86878-06-9

11th International Conference on Data Mining, Computers, Communication and Industrial Applications

(DMCCIA-2017)

Kuala Lumpur (Malaysia) Dec. 14-15, 2017

\title{
Routing Schemes for ZigBee Low-Rate Power Personal Area Network: A Survey
}

\author{
Bongisizwe E. Buthelezi, Topside E. Mathonsi, Solly Maswikaneng and Maredi Mphahlele \\ Tshwane University of Technology, Department of Information Technology, South Africa
}

\begin{abstract}
ZigBee is a Wireless Sensor Network (WSN) that was designed for Low Rate Wireless Personal Network (LR-WPAN). It is an open specification standard that was developed by ZigBee Alliance. Zigbee is the latest standard based on IEEE 802.15.4 that has recently made a lot of interest among the research community for WSN. This study gives analysis on ZigBee wireless communication protocols that could be used in Ambient Assisted Living environments such as hospitals, small clinics and homes. These environments transmit real time medical information obtained from ubiquitous non-invasive sensors to the caretaker or a doctor. This paper introduces ZigBee network, devices and the topologies supported by IEEE 802.15.4 specification. Additionally, standard Routing protocols and algorithms used in ZigBee are critically discussed based on certain metrics such as end to end delay, throughput, and packet loss.
\end{abstract}

Keywords: IEEE 802.15.4 standard, Routing algorithms, Wireless Personal Area Networks, ZigBee network.

\section{Introduction}

The usage of wireless sensor electronic devices in hospitals, small clinics, homes and in offices is currently growing exponentially. These sensory devices are used to collect real time information from the environments such as homes, hospitals and small clinics. This makes the wireless connectivity between sensory devices one of the highest priorities to network engineers and researchers. In Ambient assisted environment some of the examples of these devices are smart phones, smart televisions, body temperature, heart monitoring devices, surveillance monitoring, light controlling systems, energy consuming systems and intelligent home control systems. Homes that are equipped with these kinds of technologies are called smart homes. A smart home is one of the main applications of the Internet of things (IoT) that can be implemented as a platform, architecture, network communication, information appliance and equipment automation, centralized management and control of all kinds of household equipment, so as to create a comfortable, efficient, convenient, safe, environmentally friendly living environment [1]. Ambient Assisted Living AAL is one example of the smart home. In the smart homes we can have two kinds of networks, Wireless Personal Area Networks (WPANs) and Body Area Networks (BANs). WPANs are low power wireless personal networks built to connect devices centered on an individual person's workplace.

In December 2000 an IEEE 802 WPAN group was founded to define the wireless protocol for WPAN. WPANs are used to convey information over short distances among a private group of participant devices. ZigBee Alliance is an association of companies working together to enable reliable, cost effective, and low power wirelessly networked monitoring and control products based on an open global standard. The IEEE 802.15 working group developed personal area network consensus standards for short distance wireless personal area networks (WPANs) and the efforts of IEEE 802.15 are divided up into four main areas [2]: 


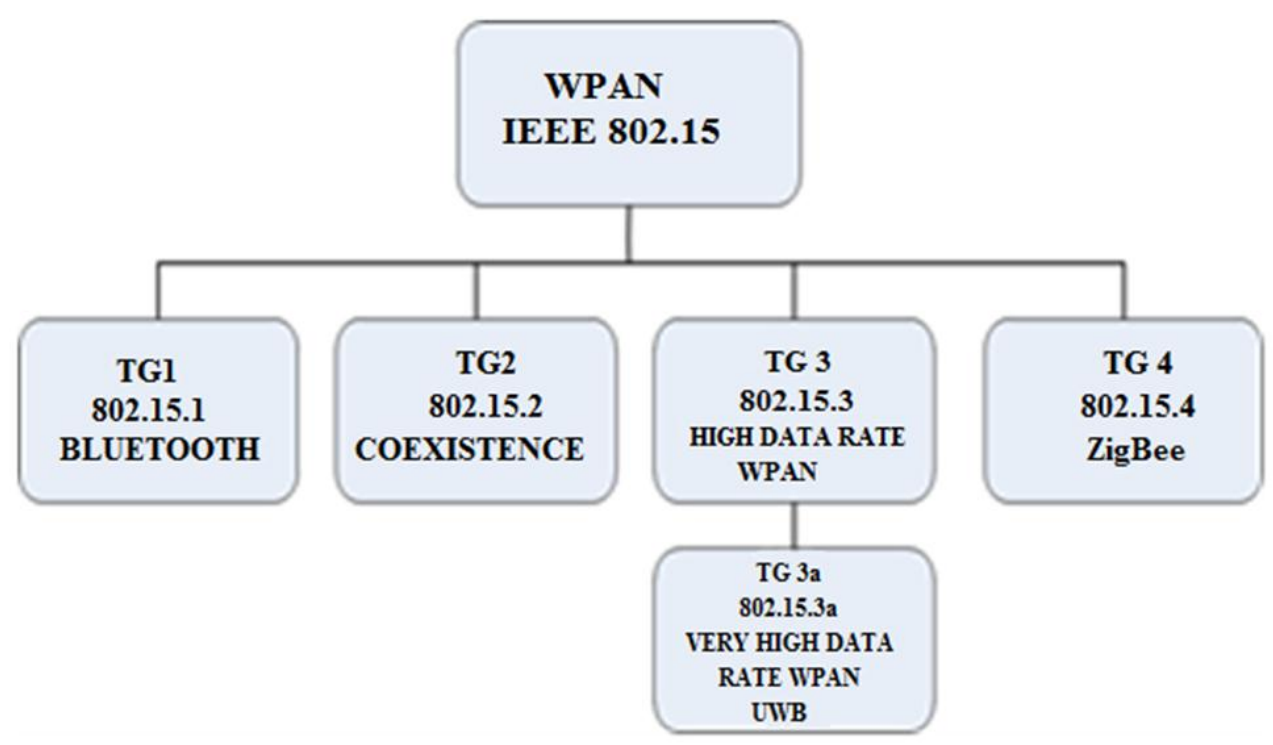

Fig.1: IEEE 802.15 Standards.

1. Task Group 1 (TG1) is creating a WPAN standard based on Bluetooth to operate in the $2.4 \mathrm{GHz}$ ISM band.

2. TG2 is concerned with the coexistence of unlicensed spectrum devices.

3. TG3 is responsible for high data rate (in excess of $20 \mathrm{Mbps)} \mathrm{WPAN} \mathrm{standards}$

4. TG4 is creating a low data rate, low power WPAN standard.

An additional group, TG3a, was created to investigate physical layer alternatives for high data rate WPAN systems

\section{Overview of The Zigbee Network}

ZigBee is WPAN communication technology that was specifically developed by ZigBee Alliance based on the 802.15.4 standard to provide low cost and low power solutions for home automation and networking [1]. Its advantage over other wireless technologies such as Bluetooth and WiFi, is its ability to ensure reliability, cost effectiveness communication, low power utilization, and its ability to enable interoperability of various products from different vendors to communicate. ZigBee uses three device types [3]: Network coordinator, Full Function Device, and Reduced Function Device. It supports star, peer to peer, and cluster tree topologies.

\section{ZigBee Network Topologies}

Compared to other PAN such as Bluetooth and WiFi, Zigbee have an advantage to support three types of topologies [3]: Star, Mesh, Tree topologies, and Cluster Tree topologies. This qualifies Zigbee to be the most suitable PAN for AAL.

Finally, its protocol architecture consists four layers namely; physical layer, media access control, network layer, and application layer. 


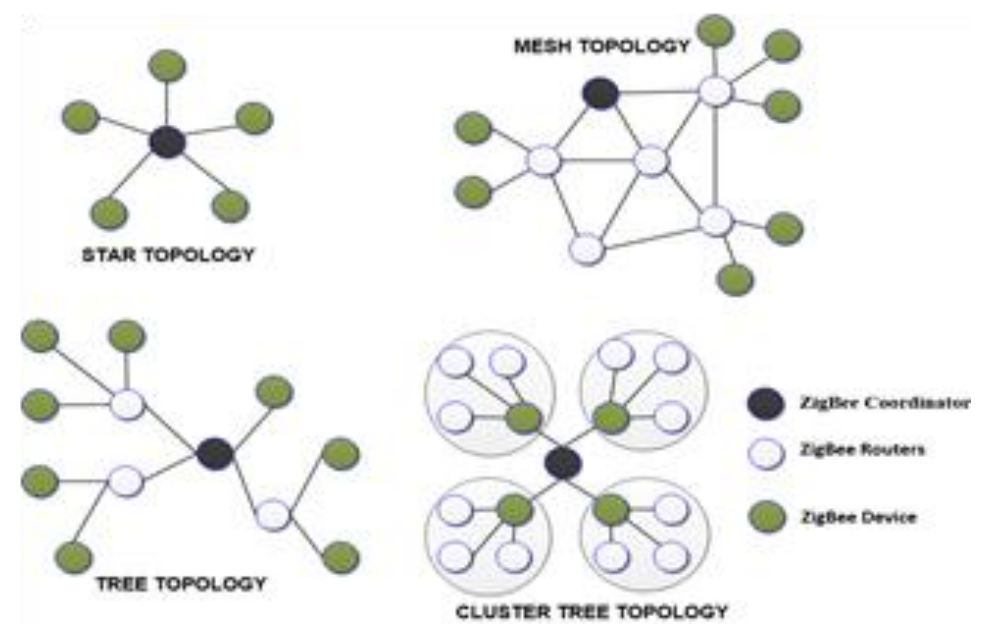

Fig.2: Types of ZigBee Topologies

\section{ZIGBEE/IEEE 802.15.4 Standard}

Based on ZigBee specification, in December 2003 a new protocol called IEEE 802.15.4 for domestic and industrial environments was released for low power and low cost wireless network. Based on the Physical (PHY) and Medium Access Control (MAC) layer, the ZigBee Alliance released its first specification in December 2004 [3]. The design of ZigBee low rate Wireless Personal Area Network (LR-WPAN)/IEEE 802.15.4 ensure reliability, low-power and cost-effective communication.

The ZigBee Protocol architecture consists of four ZigBee layers: Application layer, Network layer, Media Access Control layer, and Physical layer. Physical (PHY) layer and Media Access Control (MAC) layer are specified by IEEE 802.15.4, Network layer and Application layer are defined by ZigBee Alliance.

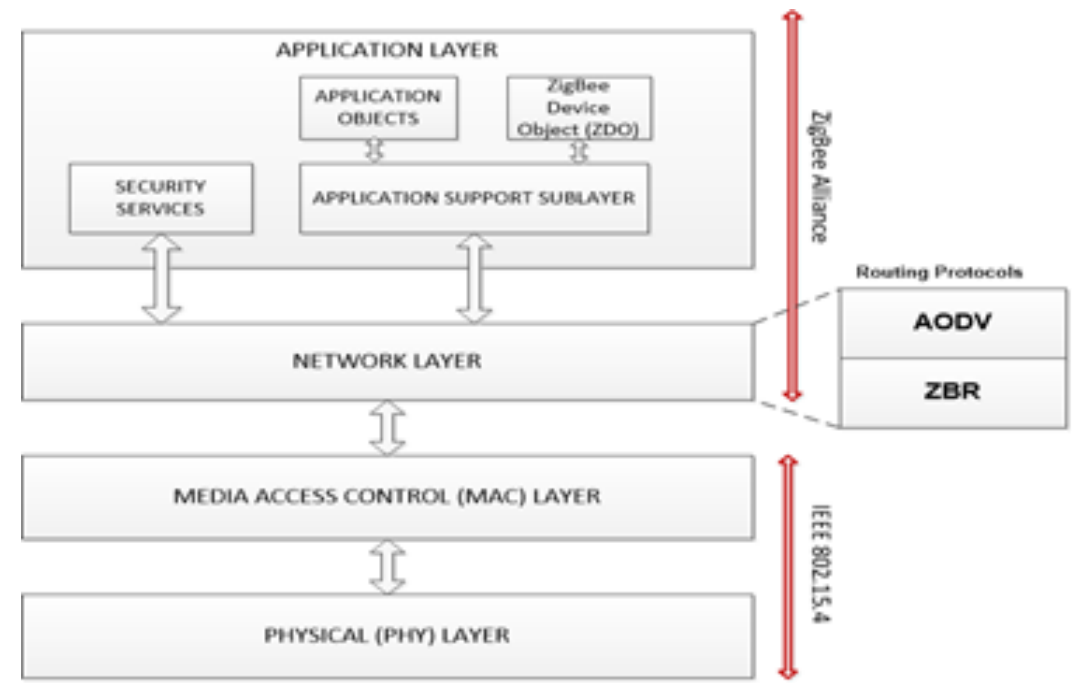

Fig.3: ZigBee/IEEE 802.15.4 Mac Layer

The Mac layer defines two types of nodes: Reduced Function Devices (RFDs) and Fully Functional Devices (FFDs) [3]. An FFD can operate in three different modes such as PAN coordinator, router, and an end device. An RFD is a device with a reduced functionality which can only be an end device. RFD does not participate in routing but it can only associate and communicate with FFDs. IEEE 802.15.4 Mac layer provide two modes of transmission: beacon enabled and non-beacon enabled. The beacon-enabled mode allows all the nodes to be time synchronized via regular beacon transmissions from the PAN-coordinator and coordinators. In the beaconenabled mode, there is no concept of regular beacon transmission and thus there is no time synchronization. The 
MAC protocol used in the beacon enabled mode is slotted Carrier Sense Multiple Access/Collision Avoidance (CSMA/CA), while unslotted CSMA/CA is used in the beacon enable mode. Moreover, the standard provides the Guaranteed Time Slots (GTS) allocation method in order to provide real time data transmission. There are three types of data transfer mechanisms between network devices: from RFD to FFD, from FFD to RFD and from FFD to FFD. Mechanisms for each transfer type depend on the existence of beacons transmission in the network.

\section{Routing Mechanism in a ZigBee Network Layer}

The ZigBee network layer defines how the network formation is performed. ZigBee specification extends the basic star topology of an IEEE 802.15.4 PAN to a cluster-tree or a mesh. In a cluster-tree topology, the root is called ZigBee coordinator (ZC) or a FFD, and all internal nodes called ZigBee routers (ZRs). RFDs can only be leaf nodes called ZigBee End Devices (ZEDs). The ZC is responsible for starting a new network whereas, the parent child relationships are established when a ZR or a ZED joins the network. When a parent (ZC or ZR) accepts a node (ZR or ZED) as its child it assigns a unique 16-bit network address to the joining node in the tree. This tree structure is also at the basis of the distributed algorithm for network address assignment [9]. A clustertree ZigBee network is characterized by topological parameters $(\mathrm{Lm}, \mathrm{Cm}, \mathrm{Rm})$.

$\boldsymbol{L m}$ : Maximum depth of the network.

$\boldsymbol{C m}$ : Maximum number of children a parent can have.

$\boldsymbol{R} \boldsymbol{m}$ : Maximum number of router children a parent can have.

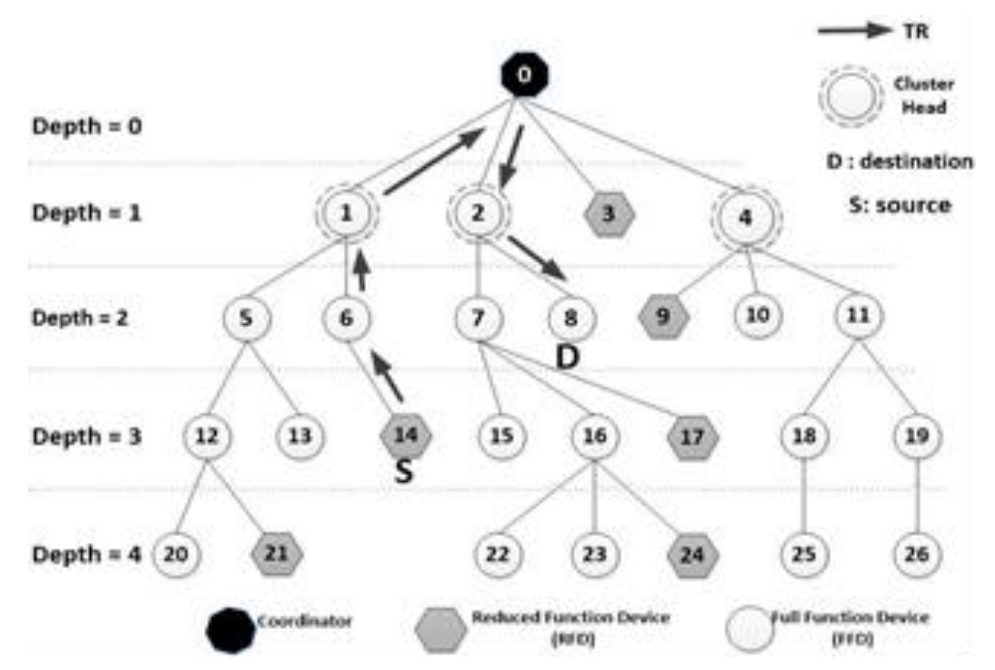

Fig.4: ZigBee Route Path on a Tree Topology.

According to ZigBee specification [3], the ZC is at depth $\mathbf{0}$ and devices at depth Lm can only be ZEDs. Let Cskip $(d), d=0,1, \ldots(L m-1)$, the size of the address sub-block being distributed by each parent at depth $\mathbf{d}$ to its router capable child devices (ZRs), is computed as follows:

$$
\operatorname{Cship}(d)=\left\{\begin{array}{l}
1+C_{m} \cdot\left(L_{m}-d-1\right), \text { if } R_{m}=1 \\
\frac{1+C_{m}-R_{m}-C_{m} \cdot R_{m}{ }_{m}^{-2 i-1}}{1-R_{m}}
\end{array}\right.
$$

where $d$ is network depth of the parent node. If the child node is a RFD, the assigned address by its parent node is:

$$
A_{n}=A_{p}+C_{s k i p}(d) \cdot R_{m}+n, 1 \leq n \leq C_{m}-R_{m}
$$

If the child node is an FFD, the address of FFD is:

$$
A_{n}=A_{p}+1+C_{\text {skip }}(d) \cdot(n-1), 1 \leq n \leq R_{m}
$$


where, $\boldsymbol{A} \boldsymbol{n}$ is an assigned address for the child node, $\boldsymbol{A} \boldsymbol{p}$ is address of the parent node.

Routing is the process of transmitting a packet from source to destination by choosing the best routes or paths in a network. Routing uses different algorithms to move packets and choose paths. Sensor network nodes are limited with respect to energy supply, restricted computational capacity and communication bandwidth. There are two basic standard types of routing algorithms used in the ZigBee network namely; AODV and the Tree Based Routing Algorithm, however there are other routing algorithms that can be implemented on a ZigBee network.

\section{Related Work}

Various routing mechanisms have been proposed for low power network called ZigBee. Recently a comprehensive study has been conducted in [4] to measure the performance of Zigbee network by comparing routing protocol such as DSR, AODV, OLSR and ZRP using NetSim Simulator. The performance parameters such as Throughput (Mbps), Delay (Microseconds), Packets Transmitted and Packets Transmitted have been analyzed. The simulation results show a better performance of AODV compared to DSR when measuring throughput. Additionally, ZRP protocol has a better result in end to end delay compared to all other routing protocols.

On demand routing protocols such as AODV, DSR and DYMO were surveyed and evaluated on a Qualnet 5.0.2 network simulator [5]. The simulation evaluation was based on throughput, average end-to-delay, and the average jitter. The output results show that DSR performs better than AODV and DYMO under network traffic load and number of hops when throughput is considered as performance metric. Additionally, in majority of the performance metrics including end to end delay DYMO has a better performance than DSR and AODV, however the mentioned mechanisms takes longer to discover the shortest routes to the destination due to the route discovery mechanisms they use.

A combination of a star-tree, star-mesh, and star-tree-mesh topologies was proposed by [6]. In this study ZigBee was implemented in a time critical Industrial Wireless Sensor Networks for Process Automation to minimize the end to end delay. The results of the proposed hybrid topology show that the Star-Tree-Mesh topology outperformed other topologies. However, this study was based on topologies whereas in our study we are more interested on routing protocols and algorithms. In [7] a new routing protocol based on Cluster-Tree algorithm was proposed by introducing a neighbor table. Their study shows more improvement in terms of minimizing end to end delay compared to an ordinary routing protocol.

Z-MHTR (ZigBee Multipath Hierarchical Tree Routing) was proposed based on simultaneous multipath routing to increase the available bandwidth in a ZigBee network [8]. Z-MHTR is a node disjoint multipath routing extension of the ZigBee tree routing protocol in cluster-tree WSNs. Simulation was performed on NS2 network simulator and the results showed that the proposed multipath routing enhances performance in terms of packet delivery ratio, end to end delay, and network lifetime.

M-HTR was proposed for the real time traffic in [9]. Firstly, this study analyzed and compared the two ZigBee routing protocols; Ad-hoc on Demand Vector (AODV) routing protocol and Hierarchical Tree Routing protocol (HTR). Secondly, HTR algorithm was modified to provide shorter delays than the original one. NS2 simulation tool was used to test the proposed M-HTR algorithm. The results of M-HTR showed improvement compared to AODV and HTR in terms of end to end delay as it provide shorter delays.

An attempt was made to measure the performance parameters of ZigBee routing protocol such as AODV and DSR using Net Sim simulator [10]. In this study it is argued that Net Sim simulator is a professional simulator that has not been used by many studies related to AODV and DSR to test and analyze the their results. Therefore authors in this study strongly argue that this was the first attempt to use this kind of a professional simulator. Performance parameters such as end to end delay, throughput, and packet delivery have been analyzed. However, based on the simulation results it shows that AODV has fewer throughputs than DSR, 
whereas DSR outperforms AODV in end to end delay and packet delivery. However, the above mentioned mechanisms ignore neighbor nodes and implement routing tables which consume energy.

An Improved Tree Routing (ImpTR) protocol determines the shortest path to the sink node depending on the neighbor table instead of following the tree topology as the original TR protocol does [11]. In ImpTR the packets are forwarded to the neighbor node if the path to the destination through neighbor node is shorter than the path through PAN coordinator. The ImpTR was evaluated on NS2 simulator, based on the results the proposed algorithm provides shorter average end to end delay, decreased energy consumption, and an increase throughput compared to the original TR routing protocol.

The hybrid routing protocol that improves the efficiency of ZigBee tree routing with a minimum additional storage and computational cost was proposed in [12]. In the proposed protocol uses the parent-child links to other one-hop neighbors if they are identified to be able to provide a route shorter than the tree path. The simulation results of the proposed protocol shows that an improvement of the routing efficiency of standard tree routing.

A region based priority mechanism was proposed to synchronize request with the Tree Routing Method on a ZigBee network [13]. Packet delivery ratio, re-transmission attempts, media access delay, queue size, queuing delay are the metric that were evaluated through simulation. The results show a better performance of the overall priority based ZigBee model than without a priority based. However, their proposed mechanism allows the packets to follow the tree based on a parent child route ignoring its neighbor nodes.

A mesh routing protocol for ZigBee called ZigBee Cluster Label (ZiCL) was proposed in [14]. ZiCL divides the ZigBee network into one or more logical clusters. The logical clusters encourage nodes to share routing information, and reduce numerical potential route discoveries. NS2 simulation was utilized for performance evaluation of the proposed model. The results show an improved network performance in terms of end to end delay and packet delivery ratio.

Based on ZigBee Cluster-Tree, an improved ZigBee Routing (ZBR) algorithm to control overhead was proposed [15]. The algorithm uses Cluster Tree parameter of ZigBee network and network addresses of the destination nodes based on two perspectives. The first one is to control the ZBR maximum transmission range and dropping the useless control overhead. The second one limits the forward packet direction of every middle route and dropping the opposite direction control overhead. The performance of the proposed algorithm was evaluated on a simulator called NS2, and the simulation results indicate the reduced control overhead and an improved overall network performance.

An algorithm called ZBR-M is an improvement of ZBR protocol which allows a horizontal exploration of the tree and more vertical exploration of the links between parent and child nodes [16]. It is argued that this proposed algorithm increases the likelihood of finding of finding an alternate path from the destination without achieving a common parent node. However, this algorithm has high energy consumption compared to the original hierarchical routing. To address this limitation, [15] proposed the classification of the messages into three types, for each message type the appropriate routing protocol (AODV, ZBR or ZBR-M) is selected to use less energy and to reduce the routing cost.

\section{Conclusion}

Based on the studies above, we can conclude that there is a lot of work that has been done to improve ZigBee topologies, routing protocols and algorithms. However, we argue that there is still a room for improvement as WSN is still an emerging research area that is attracting more and more researchers and other interesting research disciplines such as Internet of Things and Artificial Intelligence. Therefore, based on this study, our future work will attempt to improve HTR protocol in order to reduce end to end delay and reduce bandwidth usage on a ZigBee network for Home Automation.

\section{Acknowledgment}


The authors would like to thank Tshwane University of Technology for financial support. The authors declare that there is no conflict of interest regarding the publication of this paper.

\section{References}

[1] S. Zhihua, "Design of smart Home System Based on ZigBee," 2016.

[2] K. R. Patel and R. Kulkarni, "Ultra-Wideband (UWB) Wireless System," 2014.

[3] ZigBee_Alliance, "ZigBee Specification," ZigBee Standards Organization, San Ramon, 2005.

[4] G. Singh and I. V. Kapoor, "Performance Evaluation of ZIGBEE Routing Protocols Using NETSIM Simulator," Advanced Research in Computer Science, vol. 8, no. 3, pp. 852-855, 2017.

[5] A. Narmada and P. S. Rao, "Performance Comparison Of Routing Protocols For Zigbee WPAN," Journal of Computer Science Issues, vol. 8, no. 6, pp. 394 - 402, 2011.

[6] S. A. Jesudurai, A. Senthilkumar and A. Puviarasu, "An Effective Data Communication Using IEEE 802.15.4 For Wireless Sensor Network," Advanced Research Trends in Engineering and Technology (IJARTET), vol. 3, no. 7, pp. 6-12, 2016.

[7] F. Meng, B. Jiang and C. Wang, "An Improvement of ZigBee Cluster-Tree Routing Protocol," Switzerland, 2012.

[8] Z. Bidai, M. Maimour and H. Haffaf, "Multipath Extension of the ZigBee Tree Routing in Cluster-Tree Wireless Sensor Networks," Mobile Computing and Multimedia Communications, vol. 4, no. 2, pp. 30-48, 2012.

[9] B. Nefzi and Y.-Q. Song, "Performance Analysis and improvement of ZigBee routing protocol," 2007.

[10] P. Nayak, Bhakare, Saraswati and N. Tabassum, "Comparative study of DSR, AODV Routing Protocol for WPAN Using NetSim Simulator," Advanced Research in Computer Science, vol. 5, no. 4, pp. 254 - 257, 2014.

[11] M. K. Al-Harbawi, M. F. A. Rasid and N. K. Noordin, "Improved Tree Routing (ImpTR) Protocol for ZigBee Network," Computer Science and Network Security, vol. 9, no. 10, pp. 146 - 152, 2009.

[12] W. Qiu, C. Qi and E. Skafidas, "A Hybrid Routing Protocol for Wireless Sensor Networks," 2007.

[13] N. Islam, M. J. H. B. Biddut, A. I. S. Swapna and M. H. R. J. Jany, "A Study on Priority Based ZigBee Network Performance Analysis with Tree Routing method," Journal of Computer and Communications,, vol. 3, pp. 1 - 10, 2015.

[14] K. K. Lee, S. H. Kim, Y. S. Choi and H. S. Park, "A Mesh Routing Protocol using Cluster Label in the ZigBee Network," 2006.

[15] J. Li, X. Zhu and N. Tang, "Study on ZigBee Network Architecture and Routing Algorithm," 2010.

[16] M. Kasraoui, A. Cabani and J. Mouzna, "ZBR-M: A New Zigbee Routing Protocol," International Journal of Computer Science and Applications, vol. 10, no. 2, pp. 15 - 32, 2013.

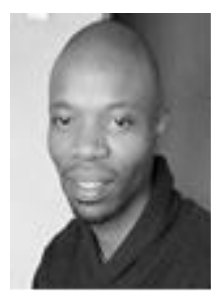

Bongisizwe Erasmus Buthelezi received his National Diploma in Information Technology majoring in Communication Networks from Mangosuthu University of Technology, South Africa, in 2010. He then went to Tshwane University of Technology, South Africa, in 2013 to pursue his BTech degree in Information Technology majoring in Communication Networks. He then became a part time lecturer in 2015 at the same department. He is currently enrolled for the MTech degree at the same department.

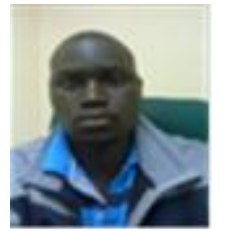

Topside E. Mathonsi received his B-Tech Information Technology (Communication Networks) in 2012 from Tshwane University of Technology, M-Tech Information Technology (Communication Networks) in 2015 and is presently studying towards his Doctor of Computing Information Technology (Communication Networks) and he is a lecturer at the Department of Information Technology at the same institution. He is a member of IAENG and a reviewer of Wireless Personal Communications journal, South African Computer Journal and Asian Journal of Computer and Information Systems. His study interests include Ad-hoc, Wireless Sensor and Network Topology (Mesh, Self-healing, Autoconfiguring). 